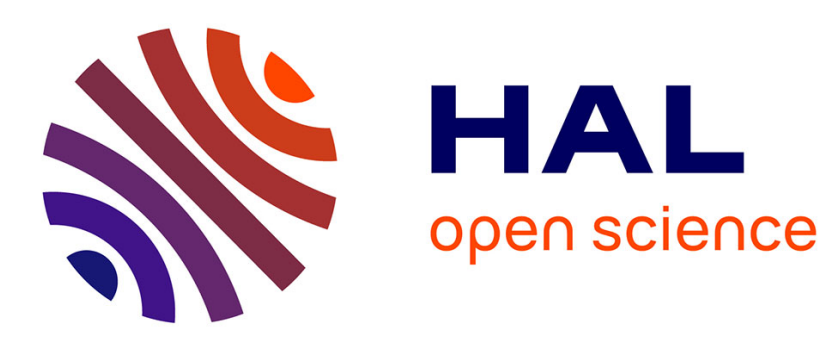

\title{
Supporting weakly Pareto optimal allocations in infinite dimensional nonconvex economies
}

Monique Florenzano, Pascal Gourdel, Alejandro Jofré

\section{To cite this version:}

Monique Florenzano, Pascal Gourdel, Alejandro Jofré. Supporting weakly Pareto optimal allocations in infinite dimensional nonconvex economies. Economic Theory, 2006, 29 (3), pp.549-564. 10.1007/s00199-005-0033-y . halshs-00086819

\section{HAL Id: halshs-00086819 \\ https://shs.hal.science/halshs-00086819}

Submitted on 21 Jul 2006

HAL is a multi-disciplinary open access archive for the deposit and dissemination of scientific research documents, whether they are published or not. The documents may come from teaching and research institutions in France or abroad, or from public or private research centers.
L'archive ouverte pluridisciplinaire HAL, est destinée au dépôt et à la diffusion de documents scientifiques de niveau recherche, publiés ou non, émanant des établissements d'enseignement et de recherche français ou étrangers, des laboratoires publics ou privés. 


\title{
SUPPORTING WEAKLY PARETO OPTIMAL ALLOCATIONS IN INFINITE DIMENSIONAL NONCONVEX ECONOMIES*
}

\author{
MONIQUE FLORENZANO ${ }^{1}$, PASCAL GOURDEL $^{2}$, AND ALEJANDRO JOFRÉ $^{3}$
}

${ }^{1}$ CNRS-CERMSEM, UMR CNRS 8095, Université Paris 1, 106-112 boulevard de l'Hôpital, 75647 Paris Cedex 13, FRANCE; monique.florenzano@univ-paris1.fr

2 CERMSEM, UMR CNRS 8095, Université Paris 1, 106-112 boulevard de l'Hôpital, 75647 Paris Cedex 13, FRANCE; gourdel@univ-paris1.fr

${ }^{3}$ Centro de Modelamiento Matematico, UMR CNRS 2071, Universidad de Chile, Blanco Encalada 2120, Casilla 170, Correo 3, Santiago, CHILE; ajofre@dim.uchile.cl

\begin{abstract}
In this paper, we prove a new version of the Second Welfare Theorem for nonconvex economies with a finite number of agents and an infinite number of commodities, that is when the preference correspondences are not convex-valued and/or when the total production set is not convex. For this kind of nonconvex economies, a recent result, obtained by one of the authors, introduces conditions which, when applied to the convex case, give for Banach commodity spaces the well-known result of decentralization by continuous prices of Pareto-optimal allocations under an interiority condition. In this paper, in order to prove a different version of the Second Welfare Theorem, we reinforce the conditions on the commodity space, assumed here to be a Banach lattice, and introduce a nonconvex version of the properness assumptions on preferences and the total production set. Applied to the convex case, our result becomes the usual Second Welfare Theorem when properness assumptions replace the interiority condition. The proof uses a HahnBanach Theorem generalization by Borwein and Jofré which allows to separate nonconvex sets in general Banach spaces.

JEL Classification numbers: D51, D6

Keywords and phrases: second welfare theorem, nonconvex economies, Banach spaces, subdifferential, Banach lattices, properness assumptions.
\end{abstract}

\section{INTRODUCTION}

For convex economies with given technological and resource limitations, individual needs and tastes, the second welfare theorem states that every Pareto optimal allocation can be supported by a non-zero price vector such that each consumer minimizes his expenditure over his preferred set and each firm maximizes its profit. Translated in mathematical terms, it means that the supporting price vector belongs to the normal cone (in the sense of convex analysis) to the total production set at the production component of the optimal allocation while its opposite belongs to the normal cone to each preferred set at the corresponding consumption component of the allocation.

Date: August 4, 2005.

*This work was partially supported by Nucleo Complex Engineering System. The successive versions of the paper were partly prepared during visits of Alejandro Jofré to CERMSEM and of Monique Florenzano and Pascal Gourdel to the Centro de Modelamiento Matematico. The hospitality of both institutions and the support of the french Coopération régionale Cone Sud are gratefully aknowledged. The authors thank Ali Khan for stimulating exchange of ideas and literature, Roko Aliprantis, Jean-Marc Bonnisseau, Alain Chateauneuf, Roger Guesnerie, Filipe Martins Da Rocha, Moncef Meddeb, B. Mordukovich, Lionel Thibault and Rabee Tourky for valuable discussions. 
In an infinite dimensional setting, that is for economies with infinitely many commodities, the result first appeared in a seminal paper of Debreu (1954) [10] where the commodity space is a topological vector space. Besides convexity hypothesis, a key assumption of Debreu's result is that the total production set of the economy has a nonempty interior, an assumption strongly related by him with free-disposal in commodities, which clearly reduces the application of the result to ordered topological vector commodity spaces whose positive orthant has a nonempty interior. A simple look to Debreu's proof, based on the Hahn-Banach theorem, allows for noticing that the interiority assumption could be likewise done for anyone of the preferred sets. However, given the assumptions currently used for guaranteeing the existence of Pareto optimal allocations, such an assumption would equally be suited for commodity spaces whose positive cone has a nonempty interior but, as previously, would prevent any application of the result to a number of commodity spaces which have been found of economic interest. This limitation explains why, thirty two years after, Debreu's result was revisited by Mas-Colell (1986) [23] who replaces Debreu's interiority condition by the so-called properness assumptions on preferences and production, at the cost of strengthening the assumptions made on the commodity space which is assumed in [23] to be a topological vector lattice and on the consumption sets restricted to coincide with the positive orthant. As in Debreu [10], the weak optimum is supported by a non-zero, continuous linear functional $p$ called "valuation functional". Moreover, $p(\omega)>0$ if the total initial endowment, $\omega>0$, is the common properness vector.

Recall that the interest for nonconvex economies, an old interest in Economics, arises on the production side from the consideration of increasing returns to scale and/or of certain externalities. On the consumption side, besides all forms of indivisibilities that we postulate away in this paper, nonconvex preferences correspond, as in Starr [31], to anti-complementarities ${ }^{1}$ between commodities, for example to non-aversion to risk for agents facing uncertainty. The reasons for considering nonconvex models in an infinite dimensional commodity space are not different. For a general study of the infinite dimensional spaces of interest in Economics, we refer the reader to the survey of Mas-Colell and Zame [24] in the Handbook of Mathematical Economics. Let us simply observe here that in a dynamic setting, technological change and the introduction of new goods and production processes should contribute to yield a nonconvex total production set. In the same way, whatever may be the formalization of uncertainty with an infinite dimensional space of states of nature (probability space or simply measurable space), the study of finance models with non convex preferences should allow to take into account the fact that a substantial fraction of the investors exhibit some uncertainty (or risk) appeal.

When the preferred sets and/or the aggregate production set are nonconvex, it is not always possible to find prices for which consumer and producer choices at a Pareto optimal allocation are truly optimal. An equilibrium notion is then defined where prices are such that choices satisfy the first order conditions for optimality written in terms of normal cones to the preferred and production sets. As well-known, even in a finite dimensional setting, several notions of normal cone have been used. Each one has a different implication for the economic significance of the decentralization result. ${ }^{2}$ But whatever be the used notion of normal cone, on the production side, the first order conditions are interpreted as pricing rules for the nonconvex firms allowing the economy as a whole to achieve efficient allocations through a mechanism that is as decentralized as

\footnotetext{
${ }^{1}$ Arrow-Hahn [4]write that "some pairs of commodities may be antagonistic in consumption".

${ }^{2}$ Think for example of Clarke's normal cone used by most of authors to study equilibrium in production with increasing returns, compared to the one used by Guesnerie [12] that can be interpreted in terms of cost minimization if the set of input combinations that produce a given level of output is convex.
} 
possible. On the consumption side, when preferences are not convex valued, rather than first order conditions, the Shapley-Folkman theorem is often used by economists to exhibit an approximate expenditure minimizing behavior (see [3] and its references). In Jofre-Rivera [18], an analogue interpretation is given using the normal cone defined in their paper.

In an infinite dimensional setting, several works on decentralization of Pareto optimal allocations have solved the question of its extension to nonconvex economies (for recent results at a level of generality comparable to Debreu [10] or Mas-Colell [23], see mainly Bonnisseau-Cornet (1988) [5], Mordukhovich (2001) [26], Jofré (2000) [17]). The three above quoted papers differ by the commodity space they consider: a topological vector space in [5], an Asplund space ${ }^{3}$ in [26], a general Banach space in [17], and the normal cone used for generalizing the normal cone of convex analysis: Clarke's normal cone in [5], Mordukhovich's normal cone in [26], Ioffe's normal cone in [17]. In view of convexity of Clarke's tangent and normal cones, the separation tool used in [5] is the Hahn-Banach theorem, while [26] and [17] use variational generalizations of the Hahn-Banach theorem, respectively an "extremal principle" due to Mordukhovich-Shao [27] and a "nonconvex separation property" due to Borwein-Jofré [6]. [17] and [26] have in common an asymptotic (net demand qualification) condition which, used jointly with the separation argument, allows to prove "viscous" or "approximate" versions of the second welfare theorem. When an additional condition, called compactly epi-Lipschitzianity, is introduced on one of the preferred or production sets then the approximate decentralization becomes exact, that is, there exists a price vector belonging to a suitable normal cone (or its opposite) to the preferred and production sets at the corresponding component of the Pareto optimal allocation. The three papers have in common ${ }^{4}$ this assumption which generalizes to the non-convex case the interiority condition of Debreu [10]. In its own setting, each one is up to now the best extension of Debreu's paper. ${ }^{5}$

The aim of this paper is to extend Mas-Colell's result to nonconvex economies defined on Banach commodity spaces. We introduce properness conditions on preferred and production sets analogue to the ones used by Mas-Colell. They can be given an analogue interpretation and allow to 'support with prices local weak Pareto optimal allocations. Properness assumptions require (in the proof of our result) a topological vector lattice structure on the commodity space and consumption sets restricted to the positive orthant, in order to use the decomposition property of vector lattices and solidness of zero neighborhoods. It then appears that properness conditions joint with a nonsatiation assumption, natural in this problem, give us for free the the asymptotic condition used in [5], [26], [17], so that we can apply the "viscous" separation property of Borwein-Jofré [6]. Also thanks to the properness assumptions, we can go from an approximate to an exact decentralization result without assuming any (compactly) epi-Lipschitzianity of one of the preferred or production sets. The supporting vector price is obtained in any normal cone satisfying some convenient properties.

It is worth noticing that preferences need not be transitive or complete and are defined for each consumer by (strictly) preferred sets. We do not make any monotonicity assumption on preferences or free-disposal assumption on production and get a decentralization result as well for production

\footnotetext{
${ }^{3}$ Asplund spaces are Banach spaces on which every continuous convex function is generically Fréchet differentiable. Particular examples are all reflexive Banach spaces and all Banach spaces with separable duals.

${ }^{4}$ In [5], compactly epi-Lipschitzianity is replaced by epi-Lipschitzianity, a stronger assumption which guarantees also that the asymptotic condition is satisfied.

${ }^{5}$ We do not include in this comparison papers of historical interest as Khan-Vohra (1988) [20] and Khan (1991) [19] which use in several respects stronger assumptions. We also omit Mordukhovich (2000) [25] which extends and slightly generalizes Jofré (2000) to several local Pareto optimality notions.
} 
economies as for pure exchange economies. As we strengthen some assumptions and weaken some other ones, this result should be considered as a complement more than a substitute to Jofré's paper. Applied to the convex case, it has as corollary the usual second welfare theorem in Banach commodity lattices when properness assumptions replace the interiority condition.

The paper is organized as follows. Section 2 recalls definitions and properties of subdifferential and normal cone which will be used later and, in order to prepare the comparison with previous results (see Remark 3.7), definitions and properties of (compactly) epi-Lipschitz sets. Section 3 describes the model, posits the main assumptions and states the main results. Section 4 is devoted to proofs.

\section{Mathematical PRELIMinaries}

Let $X$ be a Banach space, $X^{*}$ its topological dual. For $x \in X$ and $p \in X^{*}$, we note $p \cdot x$ the evaluation $p(x)$. In what follows, $B$ and $B^{*}$ denote respectively the open unit-ball of $X$ and the open unit-ball of $X^{*}, B(x, \varepsilon)$ the open ball with center $x$ and radius $\varepsilon$. Given a set $S \subset X, d_{S}(x)$ denotes the distance from $x$ to $S$ defined in the given norm by

$$
d_{S}(x):=\inf _{y \in S}\|x-y\|
$$

and

$$
\chi_{S}(x):=\left\{\begin{array}{cl}
0 & \text { if } x \in S \\
+\infty & \text { if } x \notin S
\end{array}\right.
$$

denotes the indicator function of $S$; int $S, \operatorname{cl} S$ and $\operatorname{bd} S$ denote the interior, the closure and the boundary of $S$.

If $A$ is a subset of $X^{*}, \mathrm{cl}^{*} A$ denotes the closure of $A$ for the weak-star topology $\sigma\left(X^{*}, X\right)$.

2.1. Subdifferentials and normal cones. By a subdifferential, we mean any set-valued mapping which associates with every lower semicontinuous extended-real-valued function $f$ defined on $X$ and any $x \in X$ a set $\partial f(x) \subset X^{*}$. For any $f, g X \rightarrow \mathbb{R}$ locally Lipschitz functions and any point $x \in X$, we require the following list of properties:

(a) $\partial f(x)=\emptyset$ when $x \notin \operatorname{dom}(f)$;

(b) $\partial f(x)=\partial g(x)$ if $f$ and $g$ coincide in a neighborhood of $x$;

(c) $\partial f$ is the classical subdifferential whenever $f$ is a convex function;

(d) if $f$ and $g$ are locally Lipschitz and if $x$ is a local minimum of $f+g$, then $0 \in \partial(f+g)(x) \subset$ $\partial f(x)+\partial g(x)$

(e) if $k$ is the constant of Lipschitz of $f$ near $x$, then $\|p\| \leq k$ for all $p \in \partial f(x)$;

(f) given closed subsets $Z_{1}, Z_{2}$ of $X$, the function $h: Z_{1} \times Z_{2} \rightarrow \mathbb{R}$ defined by $h\left(z_{1}, z_{2}\right)=$ $f_{1}\left(z_{1}\right)+f_{2}\left(z_{2}\right)$ satisfies $\partial h\left(z_{1}, z_{2}\right) \subset \partial f_{1}\left(z_{1}\right) \times \partial f_{2}\left(z_{2}\right)$

(g) given $A: X \rightarrow Y$, a bounded linear operator defined from a Banach space $X$ onto a Banach space $Y, A^{*}$ its adjoint, $\lambda>0$, and $b \in Y$, if $f(x)=\lambda g(A x+b)$ where $g$ is locally Lipschitz, then $\partial f(x)=\lambda A^{*} \partial g(A y+b)$.

The subdifferential is said to be robust on a class of functions if, for any function $f$ of this class, the set-valued mapping $x \rightarrow \partial f(x)$ has a closed graph in $X \times X^{*}$ when $X$ and $X^{*}$ are respectively endowed with the norm-topology and the weak-star topology $\sigma\left(X^{*}, X\right)$.

If $S \subset X$, the normal cone to $S$ at $x \in S$ associated with the given subdifferential is defined as the subdifferential of the indicator function of $S$ at $x$ :

$$
N_{S}(x)=\partial \chi_{S}(x) .
$$


That the previous list of properties, sufficient for our purposes, is nonvacuous and consistent is verified by the fact that a number of subdifferentials, in particular the approximate subdifferential, as defined by Ioffe in [16], satisfy $(\mathrm{a})-(\mathrm{g})^{6}$. Moreover, the approximate subdifferential is robust on locally Lipschitz functions, a property which plays a fundamental role in our result. If $X$ is Asplund and $f$ Lipschitz near $x$, the approximate subdifferential of $f$ at $x$ coincides with Mordukhovich's subdifferential when Mordukhovich's subdifferential is robust, in particular if $X$ is reflexive or separable (see Section 9 of [27]). If $f$ is Lipschitz near $x$, Clarke's subdifferential of $f$ at $x$ is the weak-star closed convex hull of the approximate subdifferential of $f$ at $x$ (see Theorem 7.2 of [15]).

2.2. Epi-Lipschitz sets. We recall that a subset $Z$ of a (nonnecessarily complete) normed space $X$ is called epi-Lipschitz at $z \in \operatorname{cl} Z$ with respect to $d \in X$ if there exists some $\varepsilon_{z}>0, \alpha_{z}>0$ and $\delta_{z}>0$ and a point (a direction) $d \in X$ satisfying

$$
Z \cap\left(z+\varepsilon_{z} B\right)+t\left(d+\delta_{z} B\right) \subset Z, \forall t \in\left[0, \alpha_{z}\right]
$$

A set $Z$ epi-Lipschitz at $z$ if it is epi-Lipschitz with respect to some $d$. Finally, $Z$ is epi-Lipschitz if it is epi-Lipschitz at every element $z$ in $\operatorname{cl} Z$.

The following proposition summarizes the properties of epi-Lipschitz convex sets in normed spaces.

Proposition 2.1 (Rockafellar [30]). Assume that $X$ is a normed space and $Z$ is a subset of $X$. a) If $Z$ is epi-Lipschitz at some $z \in \operatorname{cl} Z$, then $\operatorname{int} Z$ is nonempty. If, conversely, int $Z$ is nonempty and $Z$ is convex then $Z$ is epi-Lipschitz.

b) Consequently, if $Z \subset Z^{\prime}, Z$ is epi-Lipschitz at some $z \in \operatorname{cl} Z$ and $Z^{\prime}$ is convex then $Z^{\prime}$ is epi-Lipschitz.

Proof. a) is proved in [5] in the case $X$ is a topological vector space, for the corresponding notion of epi-Lipschitzianity.

The proof of $b$ ) is obvious.

We will use in this paper a "viscous" separation theorem in Banach spaces obtained by Borwein and Jofré [6] using the Ekeland variational principle and the properties (a) - (f) of the subdifferential of the distance function. We recall now its statement.

Theorem 2.1. Let $Z_{1}, \ldots, Z_{n}$ be closed subsets of a Banach space $X$ and let $\left(\bar{z}_{1}, \ldots, \bar{z}_{n}\right) \in Z_{1} \times$ $\ldots \times Z_{n}$ be such that

$$
\sum_{i=1}^{n} \bar{z}_{i} \in \operatorname{bd}\left(\sum_{i=1}^{n} Z_{i}\right)
$$

There exists $c>0$ such that for any $\varepsilon>0$ there exist $z_{i} \in\left(\bar{z}_{i}+\varepsilon B\right)$ and $p^{*} \in X^{*}$ such that

$$
p^{*} \in \bigcap_{i=1}^{n}\left(\partial d_{Z_{i}}\left(z_{i}\right)+\varepsilon B^{*}\right) \text { and } 0<c \leq\left\|p^{*}\right\| \leq 1 .
$$

\footnotetext{
${ }^{6}$ Proofs of the different properties for the approximate subdifferential can be found in [13] [14] [15] [16]. The reader has to be cautious with the fact that the terminology used evolves from one paper to another in the different papers of Ioffe quoted in our list of references.
} 


\section{The ECONOMIC MODEL AND RESUlTS}

Let $L$ be a Banach lattice, $L^{*}$ be its topological dual. On $L$ as commodity space, we consider an economy consisting of a finite set $M=\{1, \ldots, m\}$ of consumers and, for the sake of simplicity, one producer. Each consumer $i$ is described by a consumption set $X_{i} \subset L$, a preference correspondence $P_{i}: \prod_{k \in M} X_{k} \rightarrow X_{i}$, satisfying $x_{i} \notin P_{i}(x)$ for every $x \in \prod_{k \in M} X_{k}$. If $x \in X:=\prod_{k \in M} X_{k}, P_{i}(x)$ is interpreted as the set of those consumptions in $X_{i}$ which are (strictly) preferred by agent $i$ to $x_{i}$ when the consumption of each agent $k \neq i$ is equal to $x_{k}$. The set $Y \subset L$ is the (total) production set. The aggregate endowment is denoted by $\omega \in L_{+}$. To summarize, the economy $\mathcal{E}$ is defined by the following list:

$$
\mathcal{E}=\left(<L, L^{*}>,\left(X_{i}, P_{i}\right)_{i \in M}, Y, \omega\right) .
$$

Let $\mathcal{A}(\mathcal{E})$ be the set of all attainable allocations of the economy $\mathcal{E}$, that is:

$$
\mathcal{A}(\mathcal{E})=\left\{(x, y) \in\left(\prod_{i \in M} X_{i}\right) \times Y \mid \sum_{i \in M} x_{i}=y+\omega\right\} .
$$

In the following, $\mathcal{A}_{X}(\mathcal{E})$ will denote the projection of $\mathcal{A}(\mathcal{E})$ on $X=\prod_{i \in M} X_{i}$.

Definition 3.1. $\left(x^{*}, y^{*}\right) \in \mathcal{A}(\mathcal{E})$ is a weak Pareto optimal allocation for the economy $\mathcal{E}$ if there does not exist any $x \in \mathcal{A}_{X}(\mathcal{E})$ such that $x_{i} \in P_{i}\left(x^{*}\right)$ for all $i \in M$.

The concern of this paper is with the decentralization by prices (in a sense to be precised later) of weak locally Pareto optimal allocations whose we give now the precise definition.

Definition 3.2. $\left(x^{*}, y^{*}\right) \in \mathcal{A}(\mathcal{E})$ is a weak locally Pareto optimal allocation for the economy $\mathcal{E}$ if for each $i=1, \ldots, m$, there exists some neighborhood $U_{i}$ of $x_{i}^{*}$ such that there does not exist any $x \in \mathcal{A}_{X}(\mathcal{E})$ satisfying $x_{i} \in\left(P_{i}\left(x^{*}\right) \cap U_{i}\right)$ for all $i \in M$.

The set of weak locally Pareto optimal allocations obviously contains the set of weak Pareto optimal allocations. When individual preferences are strictly quasi-convex, the local notions of optimality coincide with the global ones.

We thus start with a weak locally Pareto optimal allocation $\left(x^{*}, y^{*}\right)$ and make on $\mathcal{E}$ the following assumptions:

A.1: $\forall i \in M, X_{i}=L_{+}, x_{i}^{*} \in \operatorname{cl} P_{i}\left(x^{*}\right)$ and there exist $\delta_{x_{i}^{*}}>0, \lambda_{x_{i}^{*}}>0$ and $\theta_{x_{i}^{*}}>0$ such that

$$
L_{+} \cap\left(\left(P_{i}\left(x^{*}\right) \cap B\left(x_{i}^{*}, \theta_{x_{i}^{*}}\right)\right)+\Gamma_{x_{i}^{*}}\right) \subset P_{i}\left(x^{*}\right)
$$

where $\Gamma_{x_{i}^{*}}$ denotes the set $\Gamma_{x_{i}^{*}}:=\bigcup_{\left.\lambda \in] 0, \lambda_{x^{*}}\right]} \lambda\left((1 /(m+1)) \omega+\delta_{x_{i}^{*}} B\right)$;

A.2: $Y$ is norm-closed and there exist $\delta_{y^{*}}>0, \lambda_{y^{*}}>0$, and $\theta_{y^{*}}>0$ such that

$$
\forall y \in Y \cap B\left(y^{*}, \theta_{y^{*}}\right),\left(\left(y-\Gamma_{y^{*}}\right) \cap\left\{z \in L \mid z^{+} \leq y^{+}\right\}\right) \subset Y
$$

where $\Gamma_{y^{*}}$ denotes the set $\Gamma_{y^{*}}:=\bigcup_{\lambda \in] 0, \lambda_{y^{*}}} \lambda\left((1 /(m+1)) \omega+\delta_{y^{*}} B\right)$.

\section{Remarks 3.3.}

(a) The formulation of $(1 /(m+1)) \omega$-properness of preferences in Assumptions A.1 is local and close to the one given by Podczeck [28] in the convex case. It implies that $\omega>0$ and is implied by the more classical assumption

$$
L_{+} \cap\left(P_{i}\left(x_{i}^{*}\right)+K_{i}^{*}\right) \subset P_{i}\left(x_{i}^{*}\right)
$$


where $K_{i}^{*}$ is the whole open cone generated by $\Gamma_{x_{i}^{*}}$. When preferred sets are derived from a transitive and complete preference relation on consumption sets, properness at some point of the consumption set is implied by uniform properness as formulated by Mas-Colell [22]. To be restricted to consumption sets equal to the positive cone of the commodity space is a counterpart for using properness.

(b) As proved in Proposition 4.1, local properness at $x^{*}$ of preferred sets, as stated in A.1, is equivalent to the assumption that each $P_{i}\left(x^{*}\right)$ is the trace on $L_{+}$of some set, $\widehat{P}_{i}\left(x^{*}\right)$, epi-Lipschitz at $x_{i}^{*}$ with respect to $\omega$ :

$$
\forall i \in M, P_{i}\left(x^{*}\right)=\widehat{P}_{i}\left(x^{*}\right) \cap L_{+} .
$$

In the convex case, Tourky [32] states an analogous condition.

(c) In A.2, we follow (and weaken) Richard [29] for formulating the local properness of a production set at some point of this set. It is well-known that properness as defined by Richard is weaker than the definition first given by Mas-Colell [23]. Here, the local properness of $Y$ at $y^{*} \in Y$, as stated in A.2, is implied by the assumption that

$$
Y=\widehat{Y}\left(y^{*}\right) \cap Z_{y^{*}}
$$

for some set, $\widehat{Y}\left(y^{*}\right)$, epi-Lipschitz at $y^{*}$ with respect to $-\omega$, and some closed pretechnology sublattice ${ }^{7}$ of $L, Z_{y^{*}}$, comprehensive $\left(Z_{y^{*}}-L_{+}=Z_{y^{*}}\right)$, containing 0 and such that $Y \subset Z_{y^{*}}$. In the convex case, Tourky [33] states an analogous condition.

(d) The free-disposal production set $Y=-L_{+}$satisfies A.2, but $Y=\{0\}$ does not satisfies A.2. Hence the assumptions made in this paper do not cover the case of a pure exchange economy without disposal. The supportability by prices of a pure exchange economy will be established as a corollary of the main result of this paper we next present.

Theorem 3.4. Assume that $\partial$ is any subdifferential satisfying the properties $(a)-(g)$ and robust for distance functions. Let $\left(x^{*}, y^{*}\right)$ be a weak locally Pareto optimal allocation of an economy $\mathcal{E}$ satisfying at $\left(x^{*}, y^{*}\right)$ the assumptions A.1, A.2. There exists a price $p^{*} \in L^{*}$ (the topological dual of $L), p^{*} \neq 0$, such that

a) $p^{*} \in \partial d_{Y}\left(y^{*}\right)$,

b) $-p^{*} \in \bigcap_{i \in M} \partial d_{\operatorname{cl}\left(P_{i}\left(x^{*}\right)\right)}\left(x_{i}^{*}\right)$.

Moreover, $p^{*} \cdot \omega>0$.

\section{Remark 3.5.}

It is not necessary to assume as in A.1 and A.2 that $(1 /(m+1)) \omega$ is a common properness vector. Replacing $(1 / m+1) \omega$ in the definition of $\Gamma_{x_{i}^{*}}, i \in M$ and $\Gamma_{y^{*}}$ respectively by some $v_{x_{i}^{*}}>0, i \in M$ and $v_{y^{*}}>0$ and rewriting the proof of Claim 4.1 below would allow us to prove the existence of $p^{*}$ satisfying a) and b) and $p^{*} \cdot\left(\sum_{i \in M} v_{x_{i}^{*}}+v_{y^{*}}\right)>0$.

In the case of an exchange economy, we state:

Corollary 3.6. Assume that $\partial$ is any subdifferential satisfying the properties $(a)-(g)$ and robust for distance functions. Let $x^{*}$ be a weak locally Pareto optimal allocation of an economy $\mathcal{E}$ satisfying

\footnotetext{
${ }^{7} \mathrm{~A}$ slightly weaker assumption would be $Z_{y^{*}}$ is a comprehensive subset of $L$, satisfying for all $z \in Z_{y^{*}}, z^{+} \in Z_{y^{*}}$.
} 
at $x^{*}$ Assumption A.1. There exists a price $p^{*} \in L^{*}$ (the topological dual of $L$ ), $p^{*} \neq 0$, such that $-p^{*} \in \bigcap_{i \in M} \partial d_{\mathrm{cl}\left(P_{i}\left(x^{*}\right)\right)}\left(x_{i}^{*}\right)$.

Moreover, $p^{*} \cdot \omega>0$.

In the convex case, the conclusion of Theorem 3.4 means that for each consumer $i, x_{i}^{*}$ minimizes $p^{*} \cdot x_{i}$ over $\operatorname{cl}\left(P_{i}\left(x^{*}\right)\right)$, while $y^{*}$ maximizes $p^{*} \cdot y$ over the total production set $Y$. In the nonconvex case, a) and b) express the first order conditions for these optimizing behaviours. In the spirit of Debreu's terminology, $\left(x^{*}, y^{*}, p^{*}\right)$ will be called a quasi-valuation equilibrium.

\section{Remark 3.7.}

Recall that weak Pareto optimal allocations exist under several sets of assumptions on $\mathcal{E}$. For example, if each preference correspondence $P_{i}$ derives from a complete preorder on $X_{i}$ then Pareto optimal allocations exist if for some vector space topology $\sigma$ on $L, \mathcal{A}_{X}(\mathcal{E})$ is a $\sigma^{m}$-compact subset of $\prod_{i=1}^{m} X_{i}$ and the correspondences $P_{i}$ have $\sigma^{m}$-open fibers in $\prod_{i=1}^{m} X_{i} \cdot{ }^{8}$ If each preference correspondence $P_{i}$ is convex-valued then weak Pareto optimal allocations exist under the same conditions, as an immediate consequence of the Browder-Fan theorem. For an exchange economy, and if individual preferences on convex consumption sets can be represented by quasi-concave utility functions, the two previous condition can be replaced by the weaker assumption that either the "utility set" as defined by Mas-Colell in [22] or the "individually rational utility set" is a compact subset of $\mathbb{R}^{m} \cdot{ }^{9}$ As far we know, a general study of the conditions of existence of weak Pareto optimal allocations in the nonconvex and nontransitive case is missing.

In the application to infinite dimensional economic models, the vector space topology $\sigma$ on $L$ is generally thought of as the weak topology $\sigma\left(L, L^{*}\right)$ if $L$ is reflexive, the weak-star topology $\sigma(L, M)$ if $L$ has a Banach pre-dual $M$. The continuity condition put on preference correspondences is then interpreted as an impatience condition. The main difficulty is with the $\sigma^{m}$-compactness of $\mathcal{A}_{X}(\mathcal{E})$. If $L$ is an ordered Banach space and $\sigma$ a topology such that order bounded sets are bounded, boundedness of $\mathcal{A}_{X}(\mathcal{E})$ is often guaranteed by the assumption that consumption sets are bounded from below and the total production set is bounded from above. Note however that if $L$ is a Banach lattice with an order-continuous norm and if consumption sets are equal to $L_{+}, \mathcal{A}_{X}(\mathcal{E})$ is $\sigma\left(L, L^{*}\right)$-compact if $(Y+\omega) \cap L_{+}$is $\sigma\left(L, L^{*}\right)$-compact (see Theorem 4.2.4 in [1]).

This short survey of literature shows that the domain of application of decentralization results that assume epi-Lipschitzianity of one of the preferred or production sets is the same as for Debreu (54)'s result. It is restricted either to economies defined on an ordered topological vector commodity space whose positive cone has a nonempty interior or to particular examples of economies which have weak locally Pareto optimal allocation without order-boundedness assumptions on consumption and total production sets. As proved in a previous version of this paper (see [11]), the same remark holds for decentralization results which only assume the compactly epi-Lipschitzianity of some preferred or production set at the corresponding component of the allocation, as soon as, in order to guarantee the existence of weak Pareto optimal allocation, the compactly epi-Lipschitz set is also assumed to be bounded from below or bounded from above.

\footnotetext{
${ }^{8}$ For a proof, see the proof of Theorem 1.5.3 in [1].

${ }^{9}$ When the individually rational utility set is compact, it is proved in [2] that $\mathcal{E}$ has Edgeworth equilibria, thus weak Pareto optimal allocations. For nontransitive but convex valued preferences, a generalization of the Mas-Colell condition can be found in [21].
} 
In counterpart, the main limitation of our results is in the assumption that the commodity space is a Banach lattice. Supporting prices belong to the topological dual of $L$ and it is well-known that such prices may be of difficult economic interpretation if $L$ is not reflexive. A natural application is thus to nonconvex exchange or production economies defined on $L_{p}$ spaces, $1<p<\infty$. Extending our results to topological vector commodity spaces which are not topological vector lattices ${ }^{10}$ is an objective for future work.

\section{Proofs}

4.1. Characterization of properness for individual preferences. For the sake of generality,

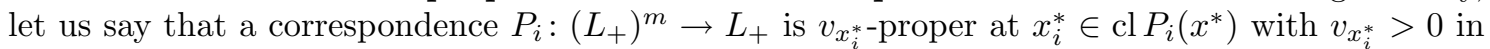
$L$ as properness vector if there exist $\delta_{x_{i}^{*}}>0, \lambda_{x_{i}^{*}}>0$ and $\theta_{x_{i}^{*}}>0$ such that

$$
L_{+} \cap\left(\left(P_{i}\left(x^{*}\right) \cap B\left(x_{i}^{*}, \theta_{x_{i}^{*}}\right)\right)+\Gamma_{x_{i}^{*}}\right) \subset P_{i}\left(x^{*}\right)
$$

where $\Gamma_{x_{i}^{*}}$ denotes the set $\Gamma_{x_{i}^{*}}:=\bigcup_{\lambda \in] 0, \lambda_{x_{i}^{*}}} \lambda\left(v_{x_{i}^{*}}+\delta_{x_{i}^{*}} B\right)$.

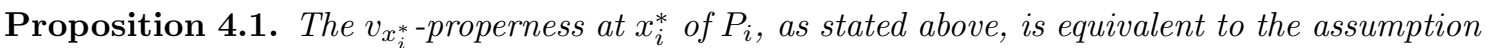
that $P_{i}\left(x^{*}\right)$ is the trace on $L_{+}$of some set, $\widehat{P}_{i}\left(x^{*}\right)$, epi-Lipschitz at $x_{i}^{*}$ with respect to $v_{x_{i}^{*}}$, that is:

$$
P_{i}\left(x^{*}\right)=\widehat{P}_{i}\left(x^{*}\right) \cap L_{+} .
$$

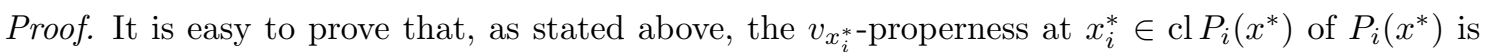
implied by the fact that $P_{i}\left(x^{*}\right)$ is the trace on $L_{+}$of some set, $\widehat{P}_{i}\left(x^{*}\right)$, epi-Lipschitz at $x_{i}^{*}$ with respect to $v_{x_{i}^{*}}$. Let us assume conversely that $P_{i}$ is $v_{x_{i}^{*}}$-proper at $x_{i}^{*} \in \operatorname{cl} P_{i}\left(x^{*}\right)$ and let us construct such set $\widehat{P}_{i}\left(x^{*}\right)$.

We can suppose that $\delta_{x_{i}^{*}} \leq\left\|v_{x_{i}^{*}}\right\| / 2$ and that $\theta_{x_{i}^{*}} \leq \lambda_{x_{i}^{*}}\left\|v_{x_{i}^{*}}\right\| / 2$. In view of this choice, we first claim that one has

$$
\Gamma_{x_{i}^{*}} \cap B\left(0, \theta_{x_{i}^{*}} / 2\right) \subset(1 / 2) \Gamma_{x_{i}^{*}} .
$$

Indeed, if $x \in \Gamma_{x_{i}^{*}} \cap B\left(0, \theta_{x_{i}^{*}} / 2\right)$, then there exist some $\left.\left.\left.\lambda \in\right] 0, \lambda_{x_{i}^{*}}\right]\right)$ and $u \in B$, such that $x=$ $\lambda\left(v_{x_{i}^{*}}+\delta_{x_{i}^{*}} u\right)$ with

$$
\left.\theta_{x_{i}^{*}} / 2\right) \geq\|x\| \geq \lambda\left(\left\|v_{x_{i}^{*}}\right\|-\delta_{x_{i}^{*}}\right) \geq \lambda\left(\left\|v_{x_{i}^{*}}\right\|\right) / 2 .
$$

Consequently, $\lambda \leq \lambda_{x_{i}^{*}} / 2$ and $x \in(1 / 2) \Gamma_{x_{i}^{*}}$.

We next define

$$
\widehat{P}_{i}\left(x^{*}\right)=P_{i}\left(x^{*}\right) \cup\left(\left(P_{i}\left(x^{*}\right) \cap B\left(x_{i}^{*}, \theta_{x_{i}^{*}} / 4\right)\right)+\Gamma_{x_{i}^{*}}\right) .
$$

In view of (4.1), $\widehat{P}_{i}\left(x^{*}\right) \cap L_{+} \subset P_{i}\left(x^{*}\right) \subset \widehat{P}_{i}\left(x^{*}\right) \cap L_{+}$, and it only remains to prove that $\widehat{P}_{i}\left(x^{*}\right)$ is epi-Lipschitz at $x_{i}^{*}$ with respect to $v_{x_{i}^{*}}$. We will prove more precisely that

$$
\left(\widehat{P}_{i}\left(x^{*}\right) \cap B\left(x_{i}^{*}, \theta_{x_{i}^{*}} / 4\right)\right)+(1 / 2) \Gamma_{x_{i}^{*}} \subset \widehat{P}_{i}\left(x^{*}\right) .
$$

Let $z \in \widehat{P}_{i}\left(x^{*}\right) \cap B\left(x_{i}^{*}, \theta_{x_{i}^{*}} / 4\right)$. If $z \in P_{i}\left(x^{*}\right)$, by definition of $\widehat{P}_{i}\left(x^{*}\right)$, the inclusion $z+(1 / 2) \Gamma_{x_{i}^{*}} \subset$ $\widehat{P}_{i}\left(x^{*}\right)$ is an obvious one. Otherwise, $z \in\left(P_{i}\left(x^{*}\right) \cap B\left(x_{i}^{*}, \theta_{x_{i}^{*}} / 4\right)\right)+\Gamma_{x_{i}^{*}}$; the point $z$ can be written as $x+\gamma$, where $x \in P_{i}\left(x^{*}\right) \cap B\left(x_{i}^{*}, \theta_{x_{i}^{*}} / 4\right)$ and $\gamma \in \Gamma_{x_{i}^{*}}$. Since $x$ and $z$ are in $B\left(x_{i}^{*}, \theta_{x_{i}^{*}} / 4\right)$, $\gamma \in B\left(0, \theta_{x_{i}^{*}} / 2\right)$ thus, in view of $(4.2), \gamma \in(1 / 2) \Gamma_{x_{i}^{*}}$, and $x+(1 / 2) \Gamma_{x_{i}^{*}} \subset \widehat{P}_{i}\left(x^{*}\right)$.

\footnotetext{
${ }^{10} \mathrm{An}$ example of space of economic interest, used for modelling commodity differentiation, is $(\mathcal{M}(K), \sigma(\mathcal{M}(K), \mathcal{C}(K)))$, the space of signed measures on a compact metric space $K$ endowed with the weakstar topology relative to its predual, the space $\mathcal{C}(K)$ of continuous real functions defined on $K$.
} 
4.2. Proof of Theorem 3.4. The proof is done in a sequence of claims that we shall establish successively. The first claim and its proof are classical in the literature devoted to properness assumptions.

Let $\delta=\min \left\{\left(\delta_{x_{i}^{*}}\right)_{i \in M}, \delta_{y^{*}}\right\}$. Using the notations of Definition 3.4, it is possible to choose some $\theta>0$ satisfying for all $i=1, \ldots, m, B\left(x_{i}^{*}, 2 \theta\right) \subset U_{i}$ and $\theta \leq \min \left\{\left(\theta_{x_{i}^{*}}\right)_{i \in M}, \theta_{y^{*}}\right\}$. Moreover, we will choose $\bar{\lambda}>0$, small enough (in particular smaller than $\min \left\{\left(\lambda_{x_{i}^{*}}\right)_{i \in M}, \lambda_{Y},\right\}$ ) such that $\Gamma \subset B(0, \theta)$, where $\Gamma$ is defined by $\Gamma=\bigcup_{\lambda \in] 0, \bar{\lambda}]}(\lambda /(m+1))(\omega+\delta B)$.

Claim 4.1. $\omega \notin \sum_{i \in M}\left(\left(P_{i}\left(x^{*}\right) \cap B\left(x_{i}^{*}, \theta\right)\right)+\Gamma\right)-\left(\left(Y \cap B\left(y^{*}, \theta\right)\right)-\Gamma\right)$.

Proof of Claim 4.1. Otherwise, there exist $x_{i} \in P_{i}\left(x^{*}\right) \cap B\left(x_{i}^{*}, \theta\right), i=1, \ldots, m, y \in Y \cap B\left(y^{*}, \theta\right)$, $u \in B, \lambda: 0<\lambda \leq \bar{\lambda}$ such that $\sum_{i \in M} x_{i}=(1-\lambda) \omega+\lambda \delta u+y$, which implies

$$
\lambda \delta u^{+} \leq \sum_{i \in M}\left(x_{i}+\lambda(1 /(m+1)) \omega\right)+y^{-}+\lambda(1 /(m+1)) \omega .
$$

Using the decomposition property of vector lattices, we have $\lambda \delta u^{+}=\sum_{i \in M} s_{i}+s_{Y}$ with for each $i \in M, 0 \leq s_{i} \leq x_{i}+\lambda(1 /(m+1)) \omega$ and $0 \leq s_{Y} \leq y^{-}+\lambda(1 /(m+1)) \omega$. For each $i \in M$, we let $x_{i}^{\prime}=x_{i}+\lambda(1 /(m+1)) \omega-s_{i}$, one has $x_{i}^{\prime} \in L_{+}$and

$$
\sum_{i \in M} x_{i}^{\prime}=\omega+y-\lambda(1 /(m+1)) \omega+\left(s_{Y}-\lambda \delta u^{-}\right) .
$$

As $\left|s_{Y}-\lambda \delta u^{-}\right| \leq s_{Y} \vee \lambda \delta u^{-} \leq \lambda \delta u^{+} \vee \lambda \delta u^{-} \leq \lambda \delta|u| \leq \lambda \delta_{y^{*}}|u|$ and $\left(y-\lambda(1 /(m+1)) \omega+\left(s_{Y}-\right.\right.$ $\left.\left.\lambda \delta u^{-}\right)\right)^{+} \leq y^{+}$, it follows from A.2 that $\sum_{i \in M} x_{i}^{\prime} \in \omega+Y$, hence that the consumption allocation $\left(x_{i}^{\prime}\right)_{i \in M} \in \mathcal{A}_{X}(\mathcal{E})$. As for each $i \in M, 0 \leq s_{i} \leq \lambda \delta u^{+} \leq \lambda \delta|u| \leq \lambda \delta_{x_{i}^{*}}|u|$ and $x_{i}^{\prime} \in L_{+}$, it follows from A.1 that for each $i \in M, x_{i}^{\prime} \in P_{i}\left(x^{*}\right)$. From our choice of $\bar{\lambda}$, for each $i \in M, x_{i}^{\prime} \in U_{i}$, which contradicts the weak local Pareto optimality of $\left(x^{*}, y^{*}\right)$.

Let us now define $\widetilde{\Gamma}=(1 / 2) \Gamma$. The two following claims are devoted to deduce from the first one that $\omega \in \operatorname{bd}\left(\sum_{i \in M} \operatorname{cl}\left(P_{i}\left(x^{*}\right) \cap B\left(x_{i}^{*}, \theta\right)\right)-\operatorname{cl}\left(Y \cap B\left(y^{*}, \theta\right)\right)+(m+1) \operatorname{cl} \widetilde{\Gamma}\right)$.

Claim 4.2. Let $\left(Q_{i}\right)_{i=1}^{m+1}$ be $\mathrm{m}+1$ nonempty subsets of $L$. Then,

$$
\operatorname{int}\left(\sum_{i=1}^{m+1} \operatorname{cl} Q_{i}+(m+1) \operatorname{cl} \widetilde{\Gamma}\right) \subset \sum_{i=1}^{m+1}\left(Q_{i}+\Gamma\right) .
$$

Proof of Claim 4.2. Indeed, let us consider $z \in \operatorname{int}\left(\sum_{i=1}^{m+1} \operatorname{cl} Q_{i}+(m+1) \operatorname{cl} \widetilde{\Gamma}\right)$. By definition of the interior, for an integer $k$ large enough, $z-(\bar{\lambda} /(2 k(m+1))) \omega \in \sum_{i=1}^{m+1} \operatorname{cl} Q_{i}+(m+1) \operatorname{cl} \widetilde{\Gamma}$. By definition of the closure, $\sum_{i=1}^{m+1} \operatorname{cl} Q_{i}+(m+1) \operatorname{cl} \widetilde{\Gamma} \subset \sum_{i=1}^{m+1} Q_{i}+(m+1) \widetilde{\Gamma}+(\bar{\lambda} \delta /(2 k(m+1))) B$. These two conditions together with the definition of $\Gamma$ imply that

$$
z \in \sum_{i=1}^{m+1} Q_{i}+(m+1) \widetilde{\Gamma}+\frac{1}{2 k} \frac{\bar{\lambda}}{(m+1)}(\omega+\delta B) \subset \sum_{i=1}^{m+1} Q_{i}+\left(m+1+\frac{1}{k}\right) \widetilde{\Gamma} .
$$

By definition of $\widetilde{\Gamma}, \sum_{i=1}^{m+1} Q_{i}+(m+1+(1 / k)) \widetilde{\Gamma} \subset \sum_{i=1}^{m+1} Q_{i}+(m+1) \Gamma=\sum_{i=1}^{m+1}\left(Q_{i}+\Gamma\right)$.

We will denote by $Q_{i}$ the set $P_{i}\left(x^{*}\right) \cap B\left(x_{i}^{*}, \theta\right)(i=1, \ldots, m)$ and by $Q_{Y}$ the set $-Y \cap B\left(y^{*}, \theta\right)$.

Claim 4.3. $\omega \in \operatorname{bd}\left(\sum_{i \in M} \operatorname{cl} Q_{i}+\operatorname{cl} Q_{Y}+(m+1) \operatorname{cl} \widetilde{\Gamma}\right)$. 
First, note that it follows from the hypothesis of local nonsatiation of preferences at each $x_{i}^{*}$, contained in A.1, that $\omega \in \sum_{i \in M} \operatorname{cl} Q_{i}+\operatorname{cl} Q_{Y}+(m+1) \operatorname{cl} \widetilde{\Gamma}$. Claim 4.1 together with Claim 4.2 applied to the sets $Q_{1}, \ldots, Q_{m}, Q_{Y}$ lead to $\omega \notin \operatorname{int}\left(\sum_{i \in M} \operatorname{cl} Q_{i}+\operatorname{cl} Q_{Y}+(m+1) \operatorname{cl} \widetilde{\Gamma}\right)$ and the conclusion holds.

Let now $\left(\varepsilon^{k}\right)$ be a sequence of positive real numbers converging to 0 . In view of Claim 4.3, we can apply Theorem 2.1 (Theorem 1 of Borwein-Jofré), in order to get for every $k$ some $p^{k} \in X^{*}$ such that

$$
\begin{gathered}
\forall i \in M,-p^{k} \in \partial d_{\mathrm{cl} Q_{i}}\left(x_{i}^{k}\right)+\varepsilon^{k} B^{*}, \\
-p^{k} \in \partial d_{-Q_{Y}}\left(-y^{k}\right)+\varepsilon^{k} B^{*}, \\
-p^{k} \in \partial d_{\mathrm{cl} \widetilde{\Gamma}}\left(z^{k}\right)+\varepsilon^{k} B^{*}, \\
0<c \leq\left\|p^{k}\right\| \leq 1
\end{gathered}
$$

where $x_{i}^{k} \in\left(x_{i}^{*}+\varepsilon^{k} B\right), y^{k} \in\left(y^{*}+\varepsilon^{k} B\right), z^{k} \in \varepsilon^{k} B$ and where $c$ does not depend on $k$. Letting $k$ tend to infinity and by passing to subnets if necessary, we get $p^{k \stackrel{\sigma\left(X^{*}, X\right)}{\longrightarrow}} p^{*}$. It then follows from the robustness of the subdifferential of the distance function that

$$
\forall i \in M,-p^{*} \in \partial d_{\mathrm{cl} Q_{i}}\left(x_{i}^{*}\right),-p^{*} \in \partial d_{-\operatorname{cl} Q_{Y}}\left(-y^{*}\right) \text { and }-p^{*} \in \partial d_{\mathrm{cl} \widetilde{\Gamma}}(0) .
$$

At this stage, it is necessary to prove that $p^{*} \neq 0$.

Claim 4.4. $p^{*} \neq 0$.

Proof. Otherwise using the decomposition $p^{k}=-u^{k}+v^{k}$, where $u^{k} \in \partial d_{\mathrm{cl}} \widetilde{\Gamma}\left(z^{k}\right)$ and $v^{k} \in \varepsilon^{k} B^{*}$, one has $u^{\sigma^{*}\left(X^{*}, X\right)} \longrightarrow$, and we will prove that it implies the norm-converges of $u^{k}$ to 0 , which contradicts that $\left\|p^{k}\right\|=\left\|-u^{k}+v^{k}\right\| \geq c>0$ since $v^{k} \in \varepsilon^{k} B^{*}$. Using condition (c) on the subdifferential, $u^{k}$ belongs to the classical normal cone of convex analysis at point $z^{k}$. This means that for all $z \in \operatorname{cl} \tilde{\Gamma}$,

$$
u^{k} \cdot\left(z-z^{k}\right) \leq 0, \text { or } u^{k} \cdot z \leq u^{k} \cdot z^{k} .
$$

Let us first remark that $u^{k} \cdot z^{k} \rightarrow 0$ since $u^{k} \in\left(1+\varepsilon^{k}\right) B^{*}$ and $z^{k} \in \varepsilon^{k} B$. By definition of $\tilde{\Gamma}$, for all $u \in B$, both $(\bar{\lambda} /(2(m+1))(\omega-\delta u)$ and $(\bar{\lambda} /(2(m+1))(\omega+\delta u)$ are in $\operatorname{cl} \Gamma$. For these points, the inequality (4.3) allows to give explicit bounds on $u^{k} \cdot u$ :

$$
\frac{1}{\delta}\left(u^{k} \cdot \omega-\frac{2(m+1)}{\bar{\lambda}} u^{k} \cdot z^{k}\right) \leq u^{k} \cdot u \leq \frac{1}{\delta}\left(\frac{2(m+1)}{\bar{\lambda}} u^{k} \cdot z^{k}-u^{k} \cdot \omega\right) .
$$

These inequalities together with the $\sigma^{*}\left(X^{*}, X\right)$ convergence of $\left(u^{k}\right)$ to 0 and our first remark lead to the norm convergence of $\left(u^{k}\right)$ to 0 and the conclusion holds.

Note that in view of the definitions of the sets $Q_{i}$ and $Q_{Y}$, it follows that the functions $d_{\mathrm{cl} Q_{i}}$ and $d_{\mathrm{cl} P_{i}\left(x^{*}\right)}$ coincide on the ball $B\left(x_{i}^{*}, \theta\right)$ and consequently using Property (b) of the subdifferential, one gets $-p^{*} \in \partial d_{\mathrm{cl} P_{i}\left(x^{*}\right)}\left(x_{i}^{*}\right)$. Finally, using the properties (b) and (g) of the subdifferential, we get: $p^{*} \in \partial d_{Y}\left(y^{*}\right)$. As $p^{*} \cdot \operatorname{cl} \widetilde{\Gamma} \geq 0$, obviously $p^{*} \cdot \omega>0$ 
4.3. Proof of Corollary 3.6. Let $\delta$ such that $0<\delta<\min \left\{\left(\delta_{x_{i}^{*}}\right)_{i \in M}\right\}$. Using the notations of Definition 3.4, it is possible to choose some $\theta>0$ satisfying for all $i=1, \ldots, m, B\left(x_{i}^{*}, 2 \theta\right) \subset U_{i}$ and $\theta \leq \min \left\{\left(\theta_{x_{i}^{*}}\right)_{i \in M}\right\}$. Moreover, we will choose $\bar{\lambda}>0$ small enough (in particular smaller than $\left.\min \left\{\left(\lambda_{x_{i}^{*}}\right)_{i \in M}\right\}\right)$ such that $\operatorname{cl} \Gamma \subset B(0, \theta)$, where $\Gamma$ denotes $\Gamma=\bigcup_{\lambda \in] 0, \bar{\lambda}]} \lambda((1 /(m+1)) \omega+\delta B)$. Note that in view of our condition on $\delta$, one has $\operatorname{cl} \Gamma \subset \Gamma_{i}^{*} \cup\{0\}$.

We will construct an auxiliary economy with production, denoted by $\mathcal{E}^{\prime}=\left(\left(X_{i}, P_{i}\right)_{i \in M}, Y^{\prime}, \omega\right)$ whose production set $Y^{\prime}$ is equal to $-\left(\operatorname{cl} \Gamma \cap L_{+}\right)$.

We first state and prove Claims 4.5 and 4.6 in order to apply Theorem 3.4 to the allocation $\left(x^{*}, 0\right)$ for the economy $\mathcal{E}^{\prime}$.

Claim 4.5. Assumption A.2 is satisfied at point $y^{*}=0$.

Proof of claim 4.5. First by construction, the set $Y^{\prime}$ is closed. We will prove that for all $y \in Y^{\prime} \cap B(0, \theta)$, if $z \in(y-(1 / 2) \Gamma) \cap\left\{z \in L \mid z^{+} \leq y^{+}\right\}$, then $z \in Y^{\prime}$.

Since $y \in Y^{\prime}, y^{+}=0$, and consequently $z^{+}=0$, which signifies $z \in-L^{+}$.

Since $z=y-\gamma$, where $y \in Y^{\prime} \cap B(0, \theta)$ and $\gamma \in(1 / 2) \Gamma$, from our choice on $\theta$, it is immediate to check that $y \in(-1 / 2) \operatorname{cl} \Gamma$ and consequently, $z \in(-1 / 2) \operatorname{cl} \Gamma-(1 / 2) \Gamma \subset-\operatorname{cl} \Gamma$.

Claim 4.6. The allocation $\left(x^{*}, 0\right)$ is a weak local Pareto optimal allocation of the economy $\mathcal{E}^{\prime}$.

Proof of claim 4.6. Indeed, otherwise there exists an allocation $\left(\left(x_{i}\right)_{i \in M}, y\right) \in \mathcal{A}(\mathcal{E})$ such that for all $i, x_{i} \in P_{i}\left(x^{*}\right) \cap B\left(x_{i}^{*}, \theta\right)$. Letting for all $i, x_{i}^{\prime}=x_{i}-(1 / m) y$, recalling that $Y^{\prime} \subset-L_{+}$, the points $x_{i}^{\prime}$ are obviously in $L_{+}$.

Since $y \in-(\operatorname{cl} \Gamma)$, one deduces that either $y=0$ or $y \in \Gamma_{i}^{*}$. If $y=0$, then $x_{i}^{\prime}=x_{i} \in P_{i}\left(x^{*}\right)$, and otherwise since $y \in \Gamma_{i}^{*}$, it follows from Assumption A.1 that $x_{i}^{\prime} \in P_{i}\left(x^{*}\right)$.

Finally by construction, one has $x_{i}^{\prime} \in B\left(x_{i}^{*}, 2 \theta\right) \subset U_{i}$ which contradicts the weak local Pareto optimality of $\left(x^{*}\right)$ in the exchange economy.

In view of Claims 4.5 and 4.6 , one can apply Theorem 3.4 to get the existence of $p^{*}$ which satisfy the conclusion of Corollary 3.6.

\section{REFERENCES}

[1] C. D. Aliprantis, D. J. Brown and O. Burkinshaw, Existence and Optimality of Competitive Equilibria, New York Heidelberg Berlin: Springer-Verlag, 1990.

[2] N. Allouch and M. Florenzano, Edgeworth and Walras equilibria of an arbitrage-free exchange economy, WP Centro de Modelamiento Matematico, CMM-B-00/12-14

[3] R. M. Anderson, The second welfare theorem with nonconvex preferences, Econometrica 56 (1988), 361-382.

[4] K. J. Arrow and F. H. Hahn, General Competitive Analysis, San Francisco: Holden-Day, INC., 1971

[5] J. M. Bonnisseau and B. Cornet, Valuation equilibrium and Pareto optimum in non-convex economies, Journal of Mathematical Economics 17 (1988), 293-308.

[6] J. M. Borwein and A. Jofré, A non-convex separation property in Banach Spaces, Journal of Operational Research and Applied Mathematics 48 (1997), 169-180.

[7] J. Borwein, Y. Lucet and B. Mordukhovich, Compactly epi-Lipschitzian convex sets and functions in normed spaces, Journal of Convex Analysis 7 No. 3 (2000), 375-393.

[8] H. Brezis, Analyse Fonctionnelle Théorie et Applications, Paris New York: Masson, 1983.

[9] F.H. Clarke, Optimization and Nonsmooth Analysis, New York: Wiley, 1983. Reprint: SIAM, 1990.

[10] G. Debreu, Valuation equilibrium and Pareto optimum, Proceedings of the National Academy of Sciences 40 (1954), 588-592.

[11] M. Florenzano, P. Gourdel and A. Jofré, Supporting weakly Pareto optimal allocations in infinite dimensional nonconvex economies, Cahiers de la MSE, srie bleue (CERMSEM) 2002.129.

[12] R. Guesnerie, Pareto optimality in non-convex economies, Econometrica 43 (1975), 1-29. 
[13] A. Ioffe, Approximate subdifferentials and applications 1. The finite dimensional theory, Transactions of the American Mathematical Society 281 No 1 (1984), 389-416.

[14] A. Ioffe, Approximate subdifferentials and applications 2. Functions on locally convex spaces, Mathematica 33 (1986), 111-128.

[15] A. Ioffe, Approximate subdifferentials and applications 3. The metric theory. Mathematica 36 (1989), 1-38.

[16] A. Ioffe, Codirectional compactness, metric regularity and subdifferential calculus, in Constructive, Experimental, and Nonlinear Analysis (M. Théra, ed.), Canadian Mathematical Society 27 (2000), 123-163.

[17] A. Jofré, A second-Welfare theorem in nonconvex economies, in Constructive, Experimental, and Nonlinear Analysis (M. Théra, ed.), Canadian Mathematical Society 27 (2000), 175-184.

[18] A. Jofré and J. R. Rivera Cayupi The second-Welfare theorem with public goods in non-convex non-transitive economies with externalities, Mimeo, Universidad de Chile (2002).

[19] M.A. Khan, Ioffe's normal cone and the foundations of Welfare economics: The infinite dimensional theory, Journal of Mathematical Analysis and Applications 161,No 1 (1991), 284-298.

[20] M.A. Khan and R. Vohra, Pareto optimal allocations of non-convex economies in locally convex spaces, Nonlinear Analysis 12, (1988), 943-950.

[21] I. Lefebvre, Application de la théorie du point fixe à une approche directe de la non-vacuité du cœur d'une économie, Thèse de Doctorat, Université de Paris 1, (2000).

[22] A. Mas-Colell, The price equilibrium existence problem in topological vector lattices, Econometrica 54 (1986), 1039-1055.

[23] A. Mas-Colell, Valuation equilibria and Pareto optimum revisited, in W. Hildenbrand and A. Mas-Colell (eds.), Contributions to mathematical economics. In honor of Gérard Debreu, Amsterdam: North-Holland (1986), 317-331.

[24] A. Mas-Colell and W. Zame, Equilibrium theory in in infinite dimensional spaces, in W. Hildenbrand and H. Sonnenschein (eds.), Handbook of Mathematical Economics, Vol. IV, Amsterdam: North-Holland (1991), $1835-1898$.

[25] B. S. Mordukhovich, An abstract extremal principle with applications to welfare economics, Journal of Mathematical Analysis and Applications 251 (2000), 187-216.

[26] B. S. Mordukhovich, The extremal principle and its applications to optimization and economics, in A. Rubinov and B. Glover (eds.), Optimization and related topics, Kluwer Academic Publishers (2001), 343-369.

[27] B. S. Mordukhovich and Y. Shao, Nonsmooth sequential analysis in Asplund spaces, Transactions of The American Mathematical Society 348 (1996), 1235-1280,

[28] K. Podczeck, Equilibria in vector lattices without ordered preferences or uniform properness, Journal of Mathematical Economics 25 (1996), 465-485.

[29] S. F. Richard, Competitive equilibria in Riesz spaces, mimeographed, GSIA, Carnegie Mellon University, 1986

[30] R. T. Rockafellar, Generalized directional derivatives and subgradients of nonconvex functions, Can. J. Math. XXXII, No. 2 (1980), 257-280.

[31] R. M. Starr, Quasi-equilibria in markets with non-convex preferences, Econometrica 37, No. 1 (1969), 25-38.

[32] R. Tourky, A new approach to the limit theorem on the core of an economy in vector lattices, Journal of Economic Theory $\mathbf{7 8}$ (1998), 321-328.

[33] R. Tourky The limit theorem on the core of a production economy with unordered preferences, Economic Theory 14 No 1 (1999), 219-226. 